\title{
New cryogenic environment for beamline ID22 at the European Synchrotron Radiation Facility
}

\author{
G. Martínez-Criado a) and R. Steinmann \\ Experiments Division, European Synchrotron Radiation Facility, 38043-Grenoble, France \\ B. Alén \\ Microelectronics Institute Madrid CNM-CSIC, 28760-Tres Cantos, Spain \\ A. Labrador \\ Laoratori de Llum de Sincrotró, BM16, European Synchrotron Radiation Facility, 38043-Grenoble, France \\ D. Fuster and J. M. Ripalda \\ Microelectronics Institute Madrid CNM-CSIC, 28760-Tres Cantos, Spain
}

\author{
A. Homs, S. Labouré, and J. Susini \\ Experiments Division, European Synchrotron Radiation Facility, 38043-Grenoble, France
}

(Received 3 October 2006; accepted 18 December 2006; published online 26 February 2007)

\begin{abstract}
A compact minicryostat has been well adapted on the hard x-ray microprobe ID22 of the European Synchrotron Radiation Facility. For variable low-temperature investigations, its special technical design provides precise scanning microscopy and allows easy access for multiple detection modes. Based on x-ray excited optical luminescence technique on the micrometer scale, details of the equipment, its temperature calibration, and typical results are described. Data collections from InAs quantum heterostructures support the excellent thermal performance of the novel cryogenic device. () 2007 American Institute of Physics. [DOI: 10.1063/1.2459375]
\end{abstract}

\section{INTRODUCTION}

The versatile instrumentation of the hard x-ray microprobe ID22 at the European Synchrotron Radiation Facility offers an excellent scheme in carrying out unique short-, medium-, and long-term research projects. ${ }^{1}$ The simultaneous scanning potentialities for trace elements analysis, quantitative fluorescence studies, chemical state characterization, and structural probes are ideal for exciting and highly demanding experiments on the micrometer scale. However, in order to perform even more challenging research and to enlarge the experimental capabilities of ID22 in the microspectroscopy domain, further instrumental developments and upgrades have been recently carried out. These include ${ }^{1}$ the possibility of carrying out $\mathrm{x}$-ray excited luminescence investigations $^{2}$ (XEOL) and (2) expanding the microscopic analysis under temperature changes using a dedicated $\mathrm{He}$ minicryostat. ${ }^{3}$ Thus, a large number of studies will be profited; particularly, those dealing with one of the most exciting and promising fields in nanotechnology.

Several important properties are very sensitive to temperature variations: (i) thermal: specific heat and thermal expansion; (ii) mechanical: elastic moduli; (iii) magnetics: superconductivity; (iv) electrical: Seebeck and Peltier effects; and (v) optical: electronic band structure. Therefore, the use of variable cryogenics has many potential applications and clear advantages for an x-ray microprobe: control thermal disorder against the static disorder, minimize radiation damage (biological samples), allow structural phase transitions

${ }^{a)}$ Electronic mail: gmartine@esrf.fr (oxides and ceramics), and permit in situ experiments at well-controlled temperatures. ${ }^{4}$

Here, we will present the development of a dedicated $\mathrm{He}$ minicryostat that suits the specific needs of the hard $\mathrm{x}$-ray microscope ID22. Based on a two undulator source, ID22 is a multipurpose beamline dedicated to $\mathrm{X}$-ray fluorescence (XRF) and x-ray absorption spectroscopy (XAS), tomography, and x-ray diffraction (XRD) studies with micron spatial resolution. After several years of refining analytical methods, hard x-ray lenses, hardware, data acquisition and processing, ID22 now offers a variety of different approaches to problems of interest in a wide range of disciplines: materials science, biology, medicine, and environmental and earth sciences.

\section{THE MINICRYOSTAT}

Figure 1(a) illustrates the typical operational setup for the system at the microprobe. In general, the mode of operation is based on a continuous flow cryostat system, with components consisting of the minicryostat, storage dewar (liquid helium), cryogen transfer tube, temperature controller, gas flow controller, and circulation pump. The liquid helium is drawn by a circulation pump from a separate cryogen storage dewar and circulated via a transfer tube through to the minicryostat heat exchanger. The cryogen flow can be regulated by a needle valve on the transfer tube. The sample is positioned on its holder which is in direct contact with the heat exchanger [Figs. 1(b) and 1(c)]. The cooling is achieved by thermal conduction from the heat exchanger to the sample holder. With the controlled flow of helium, the minicryostat 

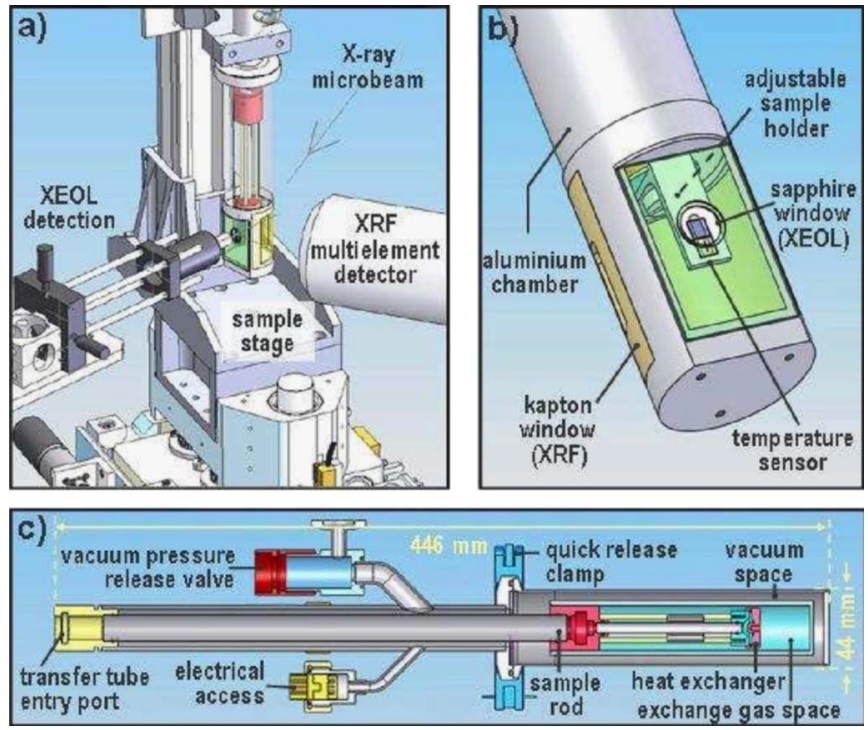

FIG. 1. (Color online) (a) Schematic layout of the minicryostat fitted with a vertical mount. (b) Amplified view of the cryostat chamber that simultaneously allows both x-ray fluorescence and x-ray excited luminescence detection modes. (c) Schematic cross-section view of the main cryostat features and dimensions.

cools down quickly $(\sim 10 \mathrm{~min}$ to $12 \mathrm{~K})$. The sample is surrounded by vacuum; in order to warm to room temperature the vacuum is released and the outer vacuum case removed. A temperature controller is used to operate and vary the temperature as required, balancing the cooling power from the cryogen and heat from the heater mounted on the heat exchanger.

The He minicryostat is adapted for microscopy applications requiring wide ranging temperatures from 300 down to $12 \mathrm{~K}$. The chamber has been specially designed and implemented to provide optimized access to the sample for multiple spectroscopic techniques [see the XRF and XEOL detection modes in Fig. 1(a)]. In general, the system has three optical windows, allowing the incident beam to impinge on the sample, luminescence and transmitted beams to be detected, as well as the x-ray emitted photons collected at $90^{\circ}$. The detailed description of the beamline is published elsewhere. ${ }^{1}$ The type of window material selected is determined by the wavelength and intensity of the radiation, the temperature, and even whether or not beam polarization is required. The mounting method minimizes strain on the windows and allows easy removal if required. Sapphire, a birefringent material, is the window used for visible and near IR applications. It is the only window which may be used up to $500 \mathrm{~K}$. At temperatures below $80 \mathrm{~K}$, the transmission of sapphire increases in the extreme IR region. This effect allows sapphire to be used as a cold inner window for IR measurements. Aluminized mylar, on the other hand, is a polyester film useful for $\mathrm{x}$-ray applications with a transmission greater than $90 \%$ and some diffuse scattering at low angles. The standard used windows are $125 \mu \mathrm{m}$ thick with a $0.3 \mu \mathrm{m}$ thick aluminum coating on one side; above $100 \mathrm{~K}$, it is porous to helium gas.

The sample holders can be used for both reflection and transmission configurations. The solid flat holder covers the
TABLE I. Summary of the relevant parameters that characterize the minicryostat.

\begin{tabular}{ll}
\hline \hline Cooling time & $9 \mathrm{~min}$ \\
Sample change time & $60 \mathrm{~min}$ \\
Temperature range & $11-300 \mathrm{~K}$ \\
Thermal stability $(\Delta T / T)$ & $<2.3 \times 10^{-2}$ at $11 \mathrm{~K}$ \\
Weight & $<0.5 \mathrm{~kg}$ \\
He consumption & $13 \mathrm{~L} /$ day at $11 \mathrm{~K}$ \\
Optical windows & Sapphire (XEOL) \\
& Mylar (XRF) \\
\hline \hline
\end{tabular}

entire optical aperture, and several small samples can be loaded simultaneously to allow multiple measurements while cold. For both fluorescence and luminescence experiments the sample holder has been designed to allow clear sample access. The maximum total angle of acceptance for reflection measurements is about $75^{\circ}$, allowing fluorescence measurements to be made at the optimal angle of the 13 -element $\mathrm{Si}$ (Li) solid state detector for most samples. In transmission, the sample may be positioned to accommodate both incident and transmitted lights over an angle of $85^{\circ}$.

High resolution small signal measurements and spatial mapping require that the mechanical stability of the cryostat does not exceed the optical spatial resolution. The mechanical $X Y Z$ translation stage, used to spot the sample at the focal point of the X-ray lens as well as at the field center of the visible light microscope, allows the components of minicryostat system to be positioned effortlessly. Interfacing plates and clamps are introduced to attach the pillared minicryostat to the $X Y Z$ stage. With the appropriate choice of the beamline slits and $\mathrm{X}$-ray focusing lens, spot sizes of less than $2 \mu \mathrm{m}^{2}$ are achieved with the entire experimental arrangement.

Therefore, with a compact and lightweight design to suit the space constraints and precise sample positioning, the introduction of the minicryostat opens up new possibilities to perform microscopic analysis. Some of the most important features are summarized in Table I. The chamber is highpure-Al made to avoid background fluorescence contribution into the collected data. To guarantee an extremely short working distance $(4.5 \mathrm{~mm})$ and optimized numerical aperture for $\mathrm{x}$-ray excited luminescence studies, the common thermal shielding used between the sample holder and the windows was not included. ${ }^{5}$ As a result, the He consumption is a little bit higher than in normal conditions. The sample change time, on the other hand, is determined by the long thermal response to warm the system up to avoid water condensation inside the chamber. The minicryostat not only allows generous access but also reduces $\mathrm{x}$-ray scattering by eliminating air path (very important for $\mathrm{x}$-ray fluorescence analysis). In addition, electrical contacts are available when transport-and/or electric-field-dependent studies are required [Fig. 1(c)].

\section{VALIDATING MEASUREMENTS}

The minicryostat offers high mechanical stability to meet the experimental microscopic needs. The beam dimensions of the achromatic focusing mirrors at the focal plane have 
Knife edge scan without cryostat
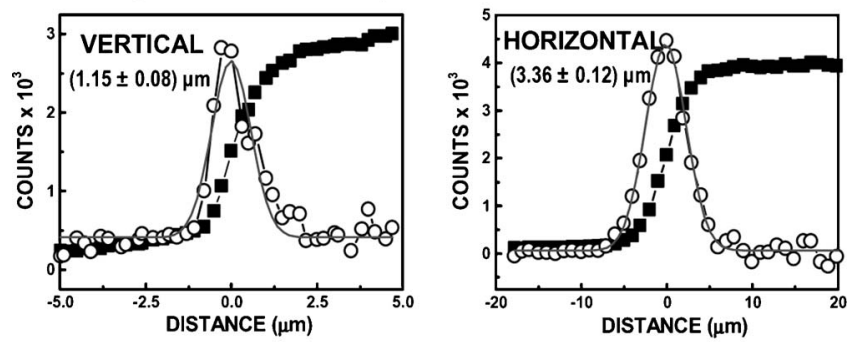

Knife edge scan at low temperature
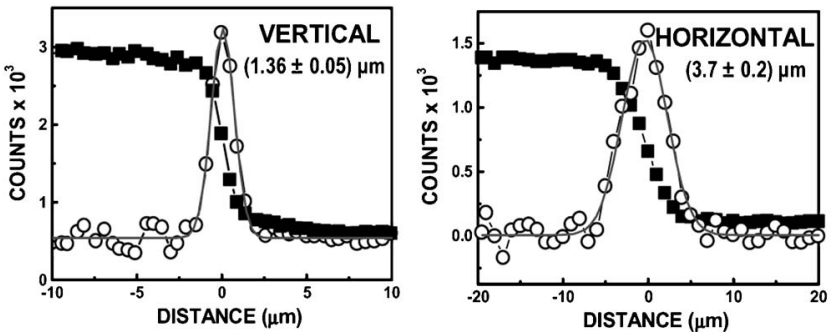

FIG. 2. Focusing beam profiles taken at $12 \mathrm{keV}$ by means of the Au knifeedge scans with and without the minicryostat.

been evaluated with and without the cryostat by the Au knife-edge scan method. ${ }^{6}$ The results taken at $12 \mathrm{keV}$ are shown in Fig. 2. These findings indicate that the mechanical stability does not exceed the optical spatial resolution. Besides, given the difference in the depth of focus between the $\mathrm{x}$-ray excited UV-visible (VIS) light and x-ray fluorescence photons, within the beamline accuracy we have found excellent performance characteristics on both collections in terms of vibrations and mechanical drifts.

For comparison reasons, using the minicryostat and also an Oxford LN2 cryostream, x-ray excited luminescence data have been collected from InAs quantum wires. Recently, an exhaustive luminescence study have been reported on their exciton recombination dynamics. ${ }^{7}$ Figure 3 clearly shows both low-temperature geometries and their corresponding re-

Oxford LN2 Cryostream 700

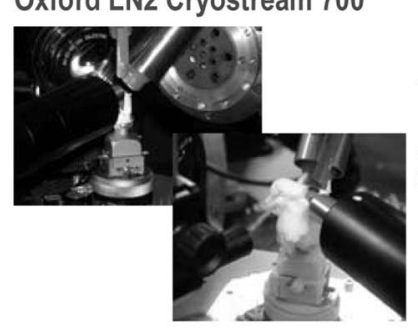

He Mini-Cryostat
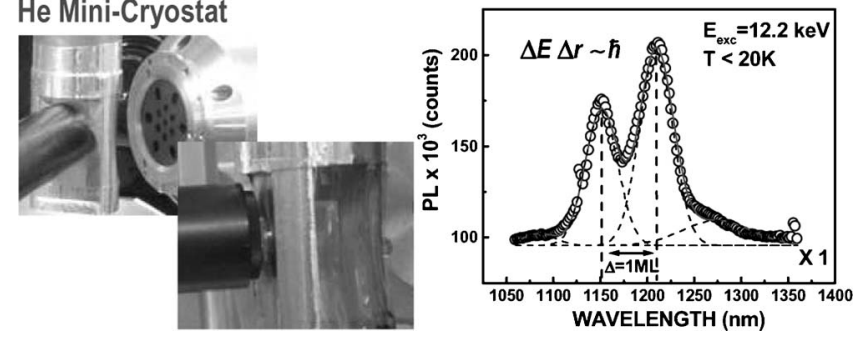

FIG. 3. X-ray excited luminescence of the InAs quantum wires taken under the liquid nitrogen cryostream and under the helium minicryostat configuration.
X-ray micro-beam - $12.2 \mathrm{keV} @ \mathrm{~T}<12 \mathrm{~K}$

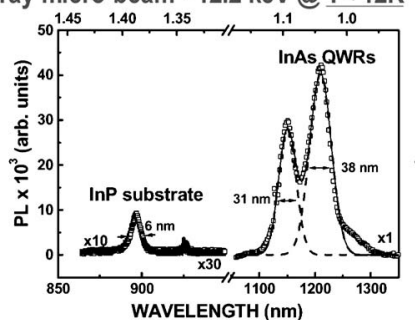

$\mathrm{Ar}^{+}$laser line - 514.5nm @ 12K
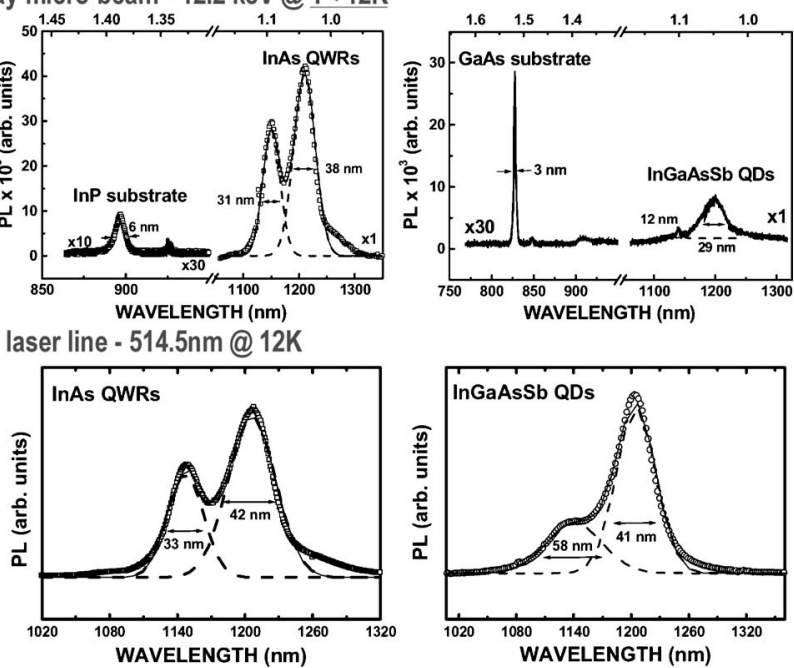

FIG. 4. Comparison between the luminescence excited by a x-ray microbeam and the luminescence excited by an Ar laser at $12 \mathrm{~K}$.

sults. With the liquid nitrogen cryostream, after long thermalization times $(>1 \mathrm{~h})$, not only is there ice formation all over the sample but there are very low count rates. While using the minicryostat (with sample in vacuum environment offering parallel reflection and transmission configurations), the intensity increases three orders of magnitude, with the radiative transitions becoming well resolved and having very sharp peaks.

Apart from the calibrated Si-diode sensor positioned on the sample holder, a complementary method in estimating the real temperature reached on the samples is through the exciton-phonon coupling itself, i.e., the linewidth of the luminescence transitions (Fig. 4), ${ }^{8}$

$$
\operatorname{FWHM}(T)=\Gamma_{o}+\gamma_{\mathrm{ac}} T+\frac{\Gamma_{\mathrm{LO}}}{e^{\hbar \omega_{\mathrm{LO}} / k_{B} T}-1} .
$$

The first term represents the broadening due to temperature-independent mechanisms (electron-electron interaction, impurity, and surface scattering), whereas the second and third terms are associated with the lifetime due to scattering by acoustic and optical phonons, respectively. Thus, taking as a reference the laser excited luminescence at $12 \mathrm{~K}$, ${ }^{9}$ the line shapes in the $\mathrm{x}$-ray excited optical luminescence [full width at half maximum $(\mathrm{FWHM})<33 \mathrm{~nm}$ ] from the InAs quantum wires and InGaAsSb quantum dots suggest that the current temperature at the sample is about $12 \mathrm{~K}$.

Thanks to the use of low temperatures and to the high thermal stability, the influence of the x-ray beam intensity itself has been evaluated through the wavelength of the intrinsic transition in GaAs substrate. The Bose-Einstein statistics describes very well the effects of the thermal expansion and of the electron-phonon interaction as a function of temperature, ${ }^{10}$

$$
E(T)=E(0)-\frac{2 \delta_{B}}{e^{\Theta_{B} / T}-1} .
$$

From the constant energy of the bound excitonic transi- 


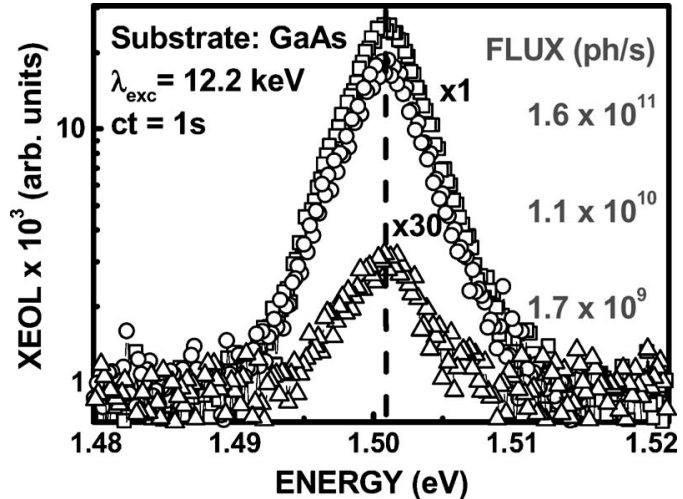

FIG. 5. Bound exciton luminescence in GaAs as a function of the x-ray photon flux.

tion in GaAs (Ref. 11) when the photon flux is changed by three orders of magnitude (Fig. 5), no heating induced by higher x-ray intensity is observed.

\section{DISCUSSIONS}

An easy to operate, miniature helium flow cryostat has been integrated at ID22 for low-temperature $\mathrm{x}$-ray microanalysis. Covering the $12-300 \mathrm{~K}$ region, with excellent mechanical stability, this simple and compact system was implemented to give highly reproducible x-ray excited luminescence and optimized collection efficiencies for x-ray fluorescence and absorption studies. The excellent performance that this novel optical cryostat can support was illustrated by the study of the excitonic lines from InAs based quantum structures on GaAs and InP substrates.

\section{ACKNOWLEDGMENTS}

Special thanks are due to Michele Soulier and Yves Dabin for the design of the mechanical interface used on the sample stage. The authors are also grateful to Remi Tucoulou as well as Isabelle Letard for their useful help.

${ }^{1}$ A. Somogyi, R. Tocoulou, G. Martínez-Criado, A. Homs, J. Cauzid, P. Bleuet, and A. Simionovici, J. Synchrotron Radiat. 12, 208 (2005).

${ }^{2}$ G. Martinez-Criado, B. Alen, A. Homs, A. Somogyi, C. Miskys, J. Susini, J. Pereira-Lachataignerais, and J. Martinez-Pastor, Appl. Phys. Lett. 89, 221913 (2006).

${ }^{3}$ R. Steinmann and P. Van der Linden, Proceedings of the International Workshop on Mechanical Engineering Design of Synchrotron Radiation Equipment and Instrumentation 2006, Egret Himeji, Japan (unpublished).

${ }^{4}$ E. I. Demikhov, Nucl. Instrum. Methods Phys. Res. A 543, 365 (2005).

${ }^{5}$ Th. Brucker, R. Nowak, Th. Kohler, U. Bruggmann, U. Maul, E. Pfutzenreuter, V. Rilling, and W. Prandl, J. Appl. Crystallogr. 29, 686 (1996).

${ }^{6}$ Y. Iketaki, Y. Horikawa, S. Mochimaru, and K. Nagai, Jpn. J. Appl. Phys., Part 1 35, 4585 (1996).

${ }^{7}$ D. Fuster, J. Martinez-Pastor, L. Gonzalez, and Y. Gonzalez, Phys. Rev. B 71, 205329 (2005).

${ }^{8}$ H. B. Bebb and E. W. Williams, Semicondcutor and Semimetals, edited by R. K. Willardson and A. C. Beer (Academic, New York, 1972), Vol. 8, p. 251.

${ }^{9}$ D. Fuster, L. Gonzalez, Y. Gonzalez, M. U. Gonzalez, and J. MartinezPastor, J. Appl. Phys. 98, 033502 (2005).

${ }^{10}$ G. Martinez-Criado, C. R. Miskys, A. Cros, A. Cantarero, O. Ambacher, and M. Stutzmann, Phys. Status Solidi B 228, 497 (2001).

${ }^{11}$ L. Pavesi and M. Guzzi, J. Appl. Phys. 75, 4779 (1994). 